\title{
SURVEY OF SELECTED PROCEDURES FOR THE INDIRECT DETERMINATION OF THE GROUP REFRACTIVE INDEX OF AIR
}

\author{
FILIP DVOŘÁČEK
}

\author{
Czech Technical University in Prague, Faculty of Civil Engineering, Department of Special Geodesy, \\ Thákurova 7, Prague, Czech Republic \\ correspondence: filip.dvoracek@fsv.cvut.cz
}

\begin{abstract}
The main aim of the research was to evaluate numeric procedures of the indirect determination of the group refractive index of air and to choose the suitable ones for requirements of ordinary and high accuracy distance measurement in geodesy and length metrology. For this purpose, 10 existing computation methods were derived from various authors' original publications and all were analysed for wide intervals of wavelengths and atmospheric parameters. The determination of the phase and the group refractive indices are essential parts in the evaluation of the first velocity corrections of laser interferometers and electronic distance meters. The validity of modern procedures was tested with respect to updated CIPM-2007 equations of the density of air. The refraction model of Leica AT401 laser tracker was analysed.
\end{abstract}

KEYWORDS: group refractive index of air; electronic distance measurement; first velocity correction; laser tracker; Leica AT401.

\section{INTRODUCTION}

In 1927 it was proposed that length etalons should be realized by the wavelength of the electromagnetic radiation instead of solid bars. Ever since then, the need for accurate determination of the refractive index of air occurred [1]. Even if a meter, the fundamental SI length unit, is today defined by the speed of light in a vacuum, the majority of measurements are performed in conditions of the lowest part of earth's atmosphere — in air.

In geodesy, length is besides angle the elementary quantity which is measured by surveying instruments and further mediates the calculation of coordinates in all Cartesian systems. During the second half of the 20th century, the electronic distance measurement (EDM) became the dominating technology in the determining both short and long distances. Nowadays, nearly all geodetic total stations enable measuring distances with coaxial EDM based on the phase or the time-of-flight (pulse) principle.

In order to correctly determine the distance between a station and a target by the EDM method, the speed of the electromagnetic radiation in ambient atmosphere has to be known. The speed of light in air is always slower than it would be in a vacuum (299 $792458 \mathrm{~m} / \mathrm{s})$ and it is significantly dependent on the state of the gaseous mixture. The physical parameter which describes the optical characteristic of the environment is called the refractive index.

Experimental procedures are employed to calculate the phase and the group refractive index of air in order to evaluate the first velocity correction of distances measured by EDM instruments. Over the years, this field of knowledge became unclear for both surveyors and instrument manufacturers. Errors and misinter- pretations can be found even in scientific papers and publications.

Please note that this article is fully focused on traditional indirect methods which determine the refractive index of air according to experiments and measurements of atmospheric parameters. The article does not cover other approaches such as a multi-wavelength interferometry, frequency comb lasers, femto-second lasers etc. which can achieve direct measurement of refractive indices [2].

\section{FundAMENTAL THEORY}

The phase refractive index $n$ of a medium is defined by a simple fraction of the speed of light in vacuum $c$ and the phase speed $v$ of the electromagnetic wave in a medium:

$$
n=\frac{c}{v} .
$$

While the phase refractive index concerns only a single wavelength $\lambda$ (e.g., for a laboratory laser interferometer), in the case of EDM instruments, a narrow band of electromagnetic waves spreads in air. Due to the phenomena called dispersion and refraction, the group speed $v_{g}$ of such a frequency band is always slower than $v$ :

$$
v_{g}=v-\frac{d v}{d \lambda} \lambda
$$

By the substitution of (1) and $c=v_{g} n_{g}$ in (2) with further arrangements and setting $n_{g} / n=1$, the group refractive index $n_{g}$ can be computed as

$$
n_{g}=n-\frac{d n}{d \lambda} \lambda .
$$


Except for the wavelength $\lambda$, the ambient group refractive index of air $n_{L}$ is dependent on conditions such as atmospheric temperature $(T, t)$, atmospheric pressure $p$ and partial water vapour pressure $e$. Changes of gaseous content of air are usually negligible except in the case of a long term drift of $\mathrm{CO}_{2}$ fraction content $x_{c}$ in atmosphere.

$$
n_{L}=f\left(\lambda, t, p, e, x_{c}\right)
$$

From an error analyses of simple procedures (IUGG 1963, IAG 1999) it can be derived that

$$
d n_{L} \cdot 10^{6}=-0.94 d t+0.28 d p-0.04 d e .
$$

Therefore the errors in parameters cause differences in the ambient group refractive index of air approximately:

$$
\begin{aligned}
& \Delta t=1{ }^{\circ} \mathrm{C} \text { causes error in } n_{L}=1.0 \mathrm{ppm} ; \\
& \Delta p=1 \mathrm{hPa} \text { causes error in } n_{L}=0.3 \mathrm{ppm} ; \\
& \Delta e=1 \mathrm{hPa} \text { causes error in } n_{L}=0.04 \mathrm{ppm} ; \\
& \Delta x_{c}=100 \mathrm{ppm} \text { causes error in } n_{L}=0.02 \mathrm{ppm} ; \\
& \Delta \lambda=1 \mathrm{~nm} \text { causes error in } n_{L}=0.02 \mathrm{ppm} .
\end{aligned}
$$

The partial water vapour pressure $e$ can be determined from a psychrometer measurement (dry and wet temperatures) or more often from the measurement of relative humidity $h$.

$$
e=\frac{E h}{100},
$$

where $E$ is the saturation water vapour pressure stated in appropriate tables or computed by Buck [3] $-E_{w}$ for $t>0{ }^{\circ} \mathrm{C}$ and $E_{i}$ for $t \leq 0{ }^{\circ} \mathrm{C}$ :

$$
\begin{array}{r}
E_{w}=\left(1+10^{-4}\left(7.2+p\left(0.0320+5.9 \cdot 10^{-6} t^{2}\right)\right)\right) \\
\cdot 6.1121^{\left(18.678-\frac{t}{234.5}\right) \frac{t}{257.14+t}} \\
E_{I}=\left(1+10^{-4}\left(2.2+p\left(0.0383+6.4 \cdot 10^{-6} t^{2}\right)\right)\right) \\
\cdot 6.1115^{\left(23.036-\frac{t}{333.7}\right) \frac{t}{279.82+t}}
\end{array}
$$

Be aware that an error in $h$ causes a greater difference in $n_{L}$ when there is a higher air temperature. For instance, $e=1 \mathrm{hPa}$ corresponds to about $h=6 \%$ at $15^{\circ} \mathrm{C}$ but only to about $h=2 \%$ at $30^{\circ} \mathrm{C}$.

For EDM instruments, a computed distance $d^{\prime}$ corresponding to reference atmospheric conditions should be adjusted to a distance $d$ which takes into account ambient atmospheric conditions:

$$
d=\left(\frac{n_{r e f}}{n_{L}}\right) d^{\prime}
$$

and therefore the first velocity correction $K^{\prime}$ can be derived as

$$
K^{\prime}=d-d^{\prime}=d^{\prime}\left(\frac{n_{r e f}-n_{L}}{n_{L}}\right) \cong d^{\prime}\left(n_{r e f}-n_{L}\right) .
$$

More basic theory can be found in [4, 5].

\section{Computation procedures}

This section shortly introduces 10 chosen procedures for computation of the group refractive index of air. Stated pieces of information are mostly gathered from the original publications of authors. The majority of symbols for variables are kept as originally stated but symbols for refractive indices are unified through the whole paper. If a method does not solve the problem of the group index, it can by computed by the derivation of the phase index and the calculation according to (3). The presentation of equations, mostly very long ones, would be hard to read in the two column style of this journal and also inadequately long for this paper. Therefore only a few chosen simplified equations are given here.

\subsection{Barrell \& Sears, 1939}

The paper [1] written by H. Barrell and J. E. Sears in 1939 was a breakthrough in the accuracy of determining of the refractive indices of air. It became the essential work in this field and many works by other authors are closely related with it. Even today, the Barrell and Sears equations are some of the best known and extensively used.

Barrell and Sears used two $670 \mathrm{~mm}$ long Fabry-Perot etalons. In the first there was a vacuum and in the second there was air at specified conditions. After the same light travelled through both etalons, they observed Brewster's circles and were able to determine the refractive index with the theoretical standard uncertainty of $5 \cdot 10^{-9}$. But the real uncertainty is considered by [5] to be about 1-2 ppm.

B\&S tested 8 wavelengths between $440 \mathrm{~nm}$ and $660 \mathrm{~nm}$ at temperatures $10-50^{\circ} \mathrm{C}$ and pressures 100 800 Torr. Dependency on the water vapour pressure was sought only at a single temperature $29.5^{\circ} \mathrm{C}$, pressure 760 Torr and relative humidity $80 \%$ and this part of the paper is considered to be the least successful. All equations are valid at $300 \mathrm{ppm}$ of $\mathrm{CO}_{2}$ content in air, the dependency on $\mathrm{CO}_{2}$ change is not given. Only the phase refractive index was being solved by the authors.

\subsection{IUGG, 1963}

In the resolution from 1963 Berkeley, International Union of Geodesy and Geodynamics (IUGG) recommended B\&S's (and Edlén's 1953) procedures. IUGG also introduced the following simplified equations derived from B\&S's procedure [5]:

$$
\begin{gathered}
\left(n_{g}-1\right) 10^{6}=287.604+3 \frac{1.6288}{\lambda^{2}}+5 \frac{0.0136}{\lambda^{4}} \\
n_{L}-1=\left(n_{g}-1\right) \frac{273.15 p}{(273.15+t) 1013.25} \\
-\frac{11.27 \cdot 10^{6}}{273.15+t} e .
\end{gathered}
$$

A modification of 12 sometimes also occurs as the following in related papers; here $\alpha$ is the coefficient of 
the thermal expansion of air, $\alpha=1 / 273.15$ :

$$
n_{L}-1=\frac{n_{g}-1}{1+\alpha t} \frac{p}{1013.25}-\frac{4.125 \cdot 10^{-8}}{1+\alpha t} e .
$$

3.3. EdLÉN, 1966

In 1953 B. Edlén evaluated several experiments of different authors (including $B \& S$ ) and published the dispersion equation for standard air [6]. In 1966 Edlén further modified the equation according to new experiments (Rank, Peck, Svensson, Erickson) [7]:

$$
(n-1) 10^{8}=8342.13+\frac{2406030}{130-\sigma^{2}}+\frac{15997}{38.9-\sigma^{2}},
$$

where $\sigma$ is the wavenumber computed as $\sigma=1 / \lambda$.

Edlén also dealt with dependency of the refractive index of air on atmospheric conditions and used Lorenz-Lorentz and air density equations. Note that both Edlén and B\&S used $f$ instead of $e$ as a symbol for the water vapour pressure [Torr]. He also dealt with the change of $x$ ppm of $\mathrm{CO}_{2}$ fractional content.

$$
\begin{gathered}
(n-1)_{x}=(1+0.540(x-0.0003))(n-1), \\
(n-1)_{t p}=\frac{p(n-1)_{x}}{720.775} \frac{1+p(0.817-0.0133 t) \cdot 10^{-6}}{1+0.0036610 t}, \\
n_{L}-n_{t p}=-f\left(5.7224-0.0457 \sigma^{2}\right) \cdot 10^{-8}
\end{gathered}
$$

Beside B\&S, Edlén's equations from 1966 are still used today in geodesy and metrology.

\subsection{OWens, 1967}

Only one year after Edlén, Owens published a paper [8] in which he examined the dependency of the refractive index on the density of air. He reached better results at high temperatures and humidities than Edlén. Important for geodesy is that special attention was given to the group refractive index. The simplified equation, which is in a great match with the full procedure is

$$
\begin{gathered}
\left(n_{g}-1\right) \cdot 10^{8}=\left(2371.34+683939.7 \frac{130+\sigma^{2}}{130-\sigma^{2}}\right. \\
\left.+4547.3 \frac{38.9+\sigma^{2}}{38.9-\sigma^{2}}\right) D_{s}+\left(6487.31+174.174 \sigma^{2}\right. \\
\left.\quad-3.55750 \sigma^{4}+0.61957 \sigma^{6}\right) D_{w}, \quad
\end{gathered}
$$

where $P_{s}$ is the pressure of dry air, $P_{w}$ is the pressure of water vapours, $P_{s}=p-P_{w}, D_{s}$ is the factor of air density, and $D_{w}$ is the factor of water vapours; the densities are given by

$$
\begin{aligned}
D_{s}= & \frac{P_{s}}{T}\left(1+P_{s}\left(57.90 \cdot 10^{-8}-\frac{9.3250 \cdot 10^{-4}}{T}\right.\right. \\
& \left.\left.+\frac{0.25844}{T^{2}}\right)\right), \quad(19) \\
D_{w}= & \frac{P_{w}}{T}\left(1+P_{w}\left(1+\left(3.7 \cdot 10^{-4}\right) P_{w}\right)\left(-2.37321 \cdot 10^{-3}\right.\right. \\
& \left.\left.+\frac{2.23366}{T}-\frac{710.792}{T^{2}}+\frac{7.75141 \cdot 10^{4}}{T^{3}}\right)\right) .
\end{aligned}
$$

\subsection{PECK \& READER, 1972}

Peck \& Reader performed measurements at 8 different wavelengths in the infrared spectrum and discovered important differences from the Edlén's 1966 formula. They also found error data of Peck \& Khanna which Edlén used. In 1972 they published the dispersion equation which was valid for a very wide interval 230 $1690 \mathrm{~nm}$ of wavelength [9]. The simplified two-term dispersion formula looks as

$$
(n-1) \cdot 10^{8}=\frac{5791817}{238.0185-\sigma^{2}}+\frac{167909}{57.362-\sigma^{2}} .
$$

P\&R did not deal with the dependency of the refractive index on atmospheric parameters, but J. M. Rueger combined their quality dispersion formula with the simplified procedure of Owens and published it in [5].

\subsection{Birch \& Downs, 1994}

In 1993 Birch \& Downs reached an opinion that frequently used Edlén's equations need several corrections [10]. The reasons were the new air density formula, the refractivity of water vapours, the change of $\mathrm{CO}_{2}$ concentration and also change of the temperature scale. In 11 authors corrected their error of a constant between temperature scales IPTS-48 and ITS-90. Below, the equation for dispersion and also the equations to compute the phase refractive index at ambient temperature, atm. pressure, partial water vapour pressure and $\mathrm{CO}_{2}$ content are given:

$$
\begin{gathered}
(n-1) \cdot 10^{8}=8342.54+\frac{2406147}{130-\sigma^{2}}+\frac{15998}{38.9-\sigma^{2}}, \\
(n-1)_{x}=(1+0.540(x-0.00045))(n-1), \quad(23) \\
(n-1)_{t p}=\frac{p(n-1)_{x}}{96095.43} \frac{1+(0.601-0.00972 t) \cdot 10^{-8} p}{1+0.0036610 t} \\
n_{L}-n_{t p}=-f\left(3.7345-0.0401 \sigma^{2}\right) \cdot 10^{-10} .
\end{gathered}
$$

\subsection{IAG, 1999}

According to the recommendations of a research group established in 1991 lead by J. M. Rueger, International Association of Geodesy (IAG) published an important resolution in 1999 in Birmingham. This IAG's resolution is still today the latest of its kind concerning the refractive index of air. With this paper, obsolete equations recommended by IUGG in 1963 should no longer be used and new simplified formulas should be adopted for standard geodetic purposes up to $1 \mathrm{ppm}$ :

$$
\begin{gathered}
\left(n_{g}-1\right) \cdot 10^{6}=287.6155+\frac{4.88660}{\lambda^{2}}+\frac{0.06800}{\lambda^{4}}, \\
\left(n_{L}-1\right) \cdot 10^{6}=\frac{273.15 N_{g} p}{1013.25 T}-\frac{11.27}{T} e .
\end{gathered}
$$

Please note an interesting fact, that these simplified equations very much correspond to the IUGG 1963 
formulas. Even if IUGG did not realize it in 1963, the simplification process of Barrell \& Sears equations randomly caused a positive influence to the computation of the refractive index. Only a constant, which takes into account the $\mathrm{CO}_{2}$ concentration of $375 \mathrm{ppm}$ and changes the dispersion equation, was applied.

The simplified equations were just a minor part of the work of the research group. The main contribution was in developing a complex precision procedure of computing the refractive index of air according to state-of-the art knowledge. That is presented in the following subsection.

\subsection{Ciddor \& Hill, 1996, 1999, 2002}

The principal leaders of the specialized research group were Philip. E. Ciddor and Reginald R. Hill. They published a series of articles in the prestigious journal Applied Optics in 1996, 1999 and 2002 [12 15. Even if they did not perform their own new measurements, they gathered all important previous data and were able to combine various scientific papers to make the most complex work on this topic.

In [12] Ciddor dealt with the phase refractive index (the group index mentioned shortly) but in [13] $\mathrm{C} \& \mathrm{H}$ focused on the group refractive index specifically. There are differences between both solutions and even if the results are in congruence, the later procedure is considered physically and mathematically correct. In 14 further investigations about $\mathrm{CO}_{2}$ and dispersion equations are presented, but no significant effects on the results were observed by authors - differences less than $3 \cdot 10^{-9}$.

A program code in Fortran was developed by Ciddor \& Hill at the University of New South Wales in Australia. Since then the code has spread around the laboratories around the world and is in use e.g. at the Finnish Geospatial Research Institute (FGI) at the Nummela Standard Baseline. However it is in an old programming language and it uses only the first $\mathrm{C} \& \mathrm{H}$ paper [12] from 1996, it is usable.

It is not an elementary task to extract the computation procedure from original publications. None of the formulas are given here in the paper as there would be at least 25 equations, 26 constants and many variables to introduce. Still it is important to present the procedures for surveyors and metrologists who are interested. The minimum information needed to program the computations is available (with Czech language comments) is available online [16].

\section{Analyses of Procedures}

The main goal of the research was to analyse results from chosen computation procedures introduced in the previous section. The results of the evaluation were sorted in the form of differences from Ciddor \& Hill 1999 procedure. Please note, that Ciddor \& Hill 1999 is further marked as C\&H_99 and every other procedure is shortened analogously.

\subsection{THE CHOICE OF PARAMETERS}

Specific sets of parameters were chosen to numerically evaluate the procedures. Testing values of wavelength, air temperature, atmospheric pressure, relative humidity and $\mathrm{CO}_{2}$ fractional content had to be stated:

- wavelength $\lambda$ : 360,650, 850, $1300 \mathrm{~nm}$;

- temperature $t$ : $-40,-25,-10,5,20,35,50{ }^{\circ} \mathrm{C}$;

- pressure $p$ : 250, 500, 750, 1000, 1250, $1500 \mathrm{hPa}$;

- relative humidity $h$ : 0, 45, 75, $100 \%$;

- $\mathrm{CO}_{2}$ content $x$ : $400 \mathrm{ppm}$.

The sampling of the parameters may seem too rough, but in fact it is chosen adequately. The refractive index does not change very rapidly and even if the finer intervals were set, it would not produce any additional findings. The chosen values sufficiently represent possible measuring conditions both outdoors and in the laboratory. The interval of wavelengths covers the whole spectrum for which newer calculation procedures are stated to be valid. $650 \mathrm{~nm}$ represents common wavelength of electronic distance meters with a visible light beam, $850 \mathrm{~nm}$ then EDMs with infrared laser emitting diodes. If an equation enables the input of $\mathrm{CO}_{2}$ fractional content, $400 \mathrm{ppm}$ was used.

An objection could be that the procedures are not comparable if changes of temperature scales from IPTS-48 to IPTS-68 to EPT-76 and ITS-90 is not considered and changes are not applied to constants of older equations. Similar objection could be that the procedures are not comparable in case of exceeding stated limits of input parameters. In theory, the objections are valid. However, this comparison deals with equations such as they were developed by the authors, without further arrangements in order to improve or adapt the results. A majority of practical users of these procedures would use them without upgrades and therefore such a comparison is more reasonable for practical surveyors.

\subsection{The eVAluation of Results}

It was not reasonable to compare the results for every combination of atmospheric parameters. Therefore, only a single parameter type was set to be a variable and all others were set as fixed. For example for testing the influence of temperature at a specific wavelength, atmospheric pressure was fixed to $1000 \mathrm{hPa}$ and relative humidity to $0 \%$. Numerical computation were performed in the Mathworks Matlab system and results were automatically exported into Microsoft Excel spreadsheet editor for further processing.

As there are a great amount of output tables and graphs, only two representatives are given here. The first is Table 1 showing the influence of changing temperature at the $360 \mathrm{~nm}$ wavelength, the second is Figure 1 at the $850 \mathrm{~nm}$ wavelength concerning changeable relative humidity. It may seem too few for such an extensive comparison, but the results do not change very rapidly and more graphs and tables would be needed 


\begin{tabular}{|r|c|c|c|c|c|c|c|c|c|}
\hline \multicolumn{7}{|c|}{ Differences $10^{8}$ (n-1) CaH_99-ostatní: $\lambda=360 \mathrm{~nm}, \boldsymbol{p}=1000 \mathrm{hPa}, \boldsymbol{h}=\mathbf{0} \%, \mathrm{CO}_{2}=\mathbf{4 0 0}$ ppm } \\
\hline $\mathbf{t}\left[{ }^{\circ} \mathbf{C}\right]$ & BaS_39 & IUGG_63 & EdI_66 & Owe_67 & PaR_72 & BaD_94 & Cid_96 & IAG_99 & Cid_02 \\
\hline$-\mathbf{4 0}$ & 40,5 & $-70,8$ & $-89,6$ & $-9,8$ & 2,0 & 1,4 & $-11,9$ & $-88,1$ & 0,7 \\
\hline $\mathbf{- 2 5}$ & 25,4 & $-62,2$ & $-73,5$ & $-4,0$ & 1,2 & 0,7 & $-5,8$ & $-72,1$ & 0,5 \\
\hline $\mathbf{- 1 0}$ & 10,2 & $-56,4$ & $-61,1$ & $-0,6$ & 0,9 & 0,4 & $-2,1$ & $-59,7$ & 0,3 \\
\hline $\mathbf{5}$ & $-4,9$ & $-52,6$ & $-51,4$ & 1,1 & 0,8 & 0,3 & $-0,3$ & $-50,1$ & 0,2 \\
\hline $\mathbf{2 0}$ & $-20,0$ & $-50,3$ & $-43,8$ & 1,4 & 0,8 & 0,4 & 0,2 & $-42,6$ & 0,1 \\
\hline $\mathbf{3 5}$ & $-35,0$ & $-49,2$ & $-38,0$ & 0,8 & 0,7 & 0,3 & $-0,2$ & $-36,8$ & 0,1 \\
\hline $\mathbf{5 0}$ & $-50,1$ & $-49,0$ & $-33,4$ & $-0,6$ & 0,6 & 0,2 & $-1,4$ & $-32,3$ & 0,0 \\
\hline
\end{tabular}

TABLE 1. $360 \mathrm{~nm}$ : Comparison of methods for changeable air temperature.

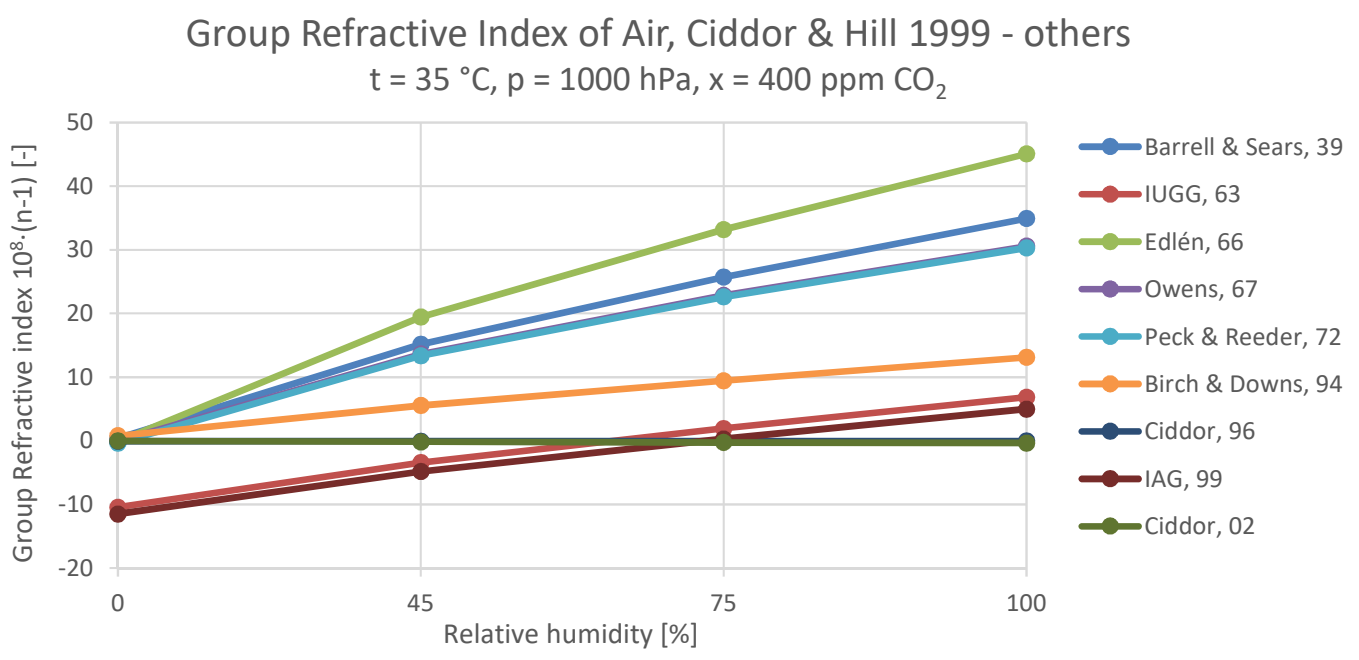

Figure 1. $850 \mathrm{~nm}$ : Comparison of methods for changeable relative humidity.

only for some specific scientific tasks. In that case, the freely available DRefraction software (Section 4.4) enables to quickly mediate an accurate comparison of procedures according to required settings.

For all the wavelengths and all the tested atmospheric parameters, the differences between the reference $\mathrm{C} \& \mathrm{H} \_99$ and both $\mathrm{C} \& \mathrm{H} \_96$ and $\mathrm{C} \& \mathrm{H} \_02$ procedures are low, less than $1 \cdot 10^{-8}$. It is in congruence with the statements of Ciddor and Hill. Such differences would not significantly influence measurements performed by surveyors.

The $360 \mathrm{~nm}$ wavelength is the lowest limit of validity for some newer procedures. Equations derived from the original B\&S_39 observations (B\&S_39, IUGG_63 and IAG_99) are the most inconsistent ones in the case of changeable temperature and pressure values (up to $0.9 \mathrm{ppm}$ ). For changeable relative humidity, it stands that newer equations are better that older ones and differences exceed even $1 \mathrm{ppm}$.

The most important tables and graphs concern 650 $\mathrm{nm}$ and $850 \mathrm{~nm}$ wavelength because these parts of electromagnetic radiation are frequently used in geodesy. Even if older procedures were not valid for infrared wavelengths, it showed that they are still quite usable for purposes of ordinary measurement. All results are within $\pm 0.2 \mathrm{ppm}$ interval (for ordinary conditions even within $\pm 0.1 \mathrm{ppm}$ interval) for changeable temperature and pressure. For changeable humidity, differences are according to expectations greater, up to $0.5 \mathrm{ppm}$ (e.g., Edl_66). For IUGG_63 and IAG_99 it is obvious that the results are better than in the case of the original BaS_39 procedure. Simplified equations IAG_99 are reliable up to $0.1 \mathrm{ppm}$ from the complex C\&H_99 procedure.

Even for the $1300 \mathrm{~nm}$ wavelength, the older equations are still usable with similar results as for $850 \mathrm{~nm}$. The extrapolation of equations is much more reliable within the infrared spectrum than in the direction to the ultraviolet spectrum because there is an uncomplicated dispersion dependency.

\subsection{C\&H AND THE NEW CIPM-2007 EQUATION}

Ciddor \& Hill based their calculations on equations of air density according to Giacomo [17] and Davis [18. The most important equation is usually called the CIMP-81/91. In 2008 the equation was revised by a group of authors and it was called CIPM-2007 formula 19. A larger molar fraction of argon and a new molar gas constant were employed:

$$
R=8.31447215 \mathrm{~J} \mathrm{~mol}^{-1} \mathrm{~K}
$$




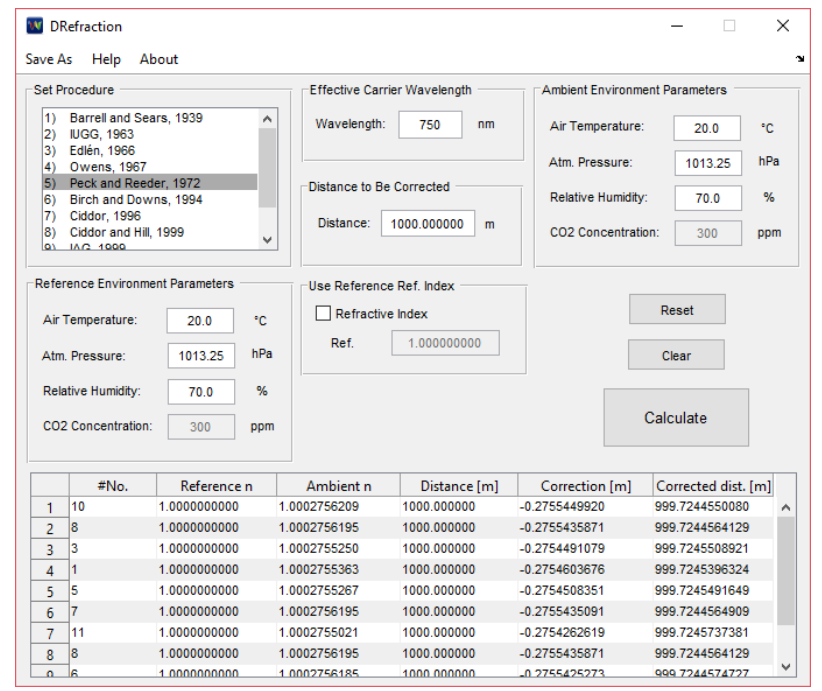

Figure 2. Graphical user interface of the DRefraction software.

therefore also a change in the calculation of the molar weight of dry air was carried out:

$$
M_{a}=10^{-3}\left(28.96546+12.011 \cdot 10^{-6}\left(x_{c}-400\right)\right) \frac{\mathrm{kg}}{\operatorname{mol}} .
$$

Other constants were left unchanged.

It should be verified whether these new pieces of knowledge influence the computation of the refractive index of air according to $\mathrm{C} \& \mathrm{H}$ procedures. The author of this paper numerically checked that at the 1e-9 level there is no change in the results and, therefore, no adjustment of the procedure is needed. In this case, the continuity of the existing equations and subsequent numerical outputs are more important than the precise agreement with physical constants.

\subsection{DREFRACTION SOFTWARE}

As all the discussed procedures from section 3 were adopted and programmed in the Mathworks Matlab system, it was convenient to prepare also a software with a graphical user interface. The application is called DRefraction (Fig. 2) and it is freely available for downloading for 64bit Windows PCs. The group refractive index can be easily computed according to a chosen computation procedure. Both the reference atmospheric conditions and the reference refractive index can be used to compute the first velocity correction for electronic distance meters. Results can be exported into txt file for further processing. If required, the software can be easily modified in order to compute also the phase refractive indices for laser interferometers. The phase refractive index of air of Ciddor and Hill procedures in in congruence with computations according to NIST Engineering Metrology Toolbox [20].

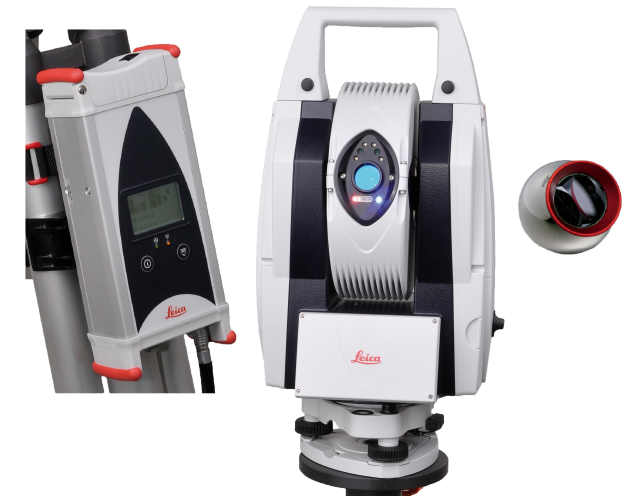

Figure 3. Leica AT401 laser tracker system.

\section{ReFraCtion MOdEL OF THE LeicA AT401}

The Leica AT401 laser tracker (Fig. 3) is an instrument whose parameters make it suitable for specific highaccuracy geodetic and metrology tasks. The maximum working range of $160 \mathrm{~m}$ and the standard deviation of $5 \mathrm{\mu m}$ (up to $40 \mathrm{~m}$ according to ASME B89.4.19-2006) for absolute distance measurement (ADM) are unique among distance meters. Compared to the state-ofthe-art total stations, the accuracy is about 100 times ( 2 orders) better. Of course, the ADM technology also has its disadvantages concerning excessive sensibility in the case of field measurement and therefore also a varying real maximum range.

The author of this paper has tested the Leica AT401 in laboratories of Research Institute of Geodesy, Topography and Cartography (RIGTC) and Czech Technical University in Prague, Faculty of Civil Engineering. The main purpose was to prepare the instrument and other equipment for high accurate field measurements at Czech national field length baselines [21].

A document (from 2013-02-28) about the refractive index of air for the Leica AT401 was acquired from the manufacturer. According to this document, the following equation is based on Edlén's formula:

$$
\begin{gathered}
N_{g}=0.2914269 P\left(\frac{1+10^{-6} P(0.613-0.010 T)}{1+0.0036610 T}\right) \\
-550.51 \cdot 10^{-6} R \cdot 10^{\frac{7.5 T}{T+237.3}+0.6609} .
\end{gathered}
$$

As the comparison of refractive methods showed in Figure 1] Edlén's equations differs the most of all others from C\&H. Considering that Leica's formula differs even more than Edl_66, Leica made an unfortunate choice. In addition, the manufacturer mixed wavelength values both in the instrument's manual and in the system software. Instead of using the correct $795 \mathrm{~nm}$ for Leica AT401, $780 \mathrm{~nm}$ was incorrectly used. (Some older laser tracker instruments used $780 \mathrm{~nm}$ ).

Besides issues mentioned above, other unexpected errors were discovered. When values of the group refractive index of air were continually observed, very few changes occurred. Further tests showed that the internal stored value of the refractive index is changed 


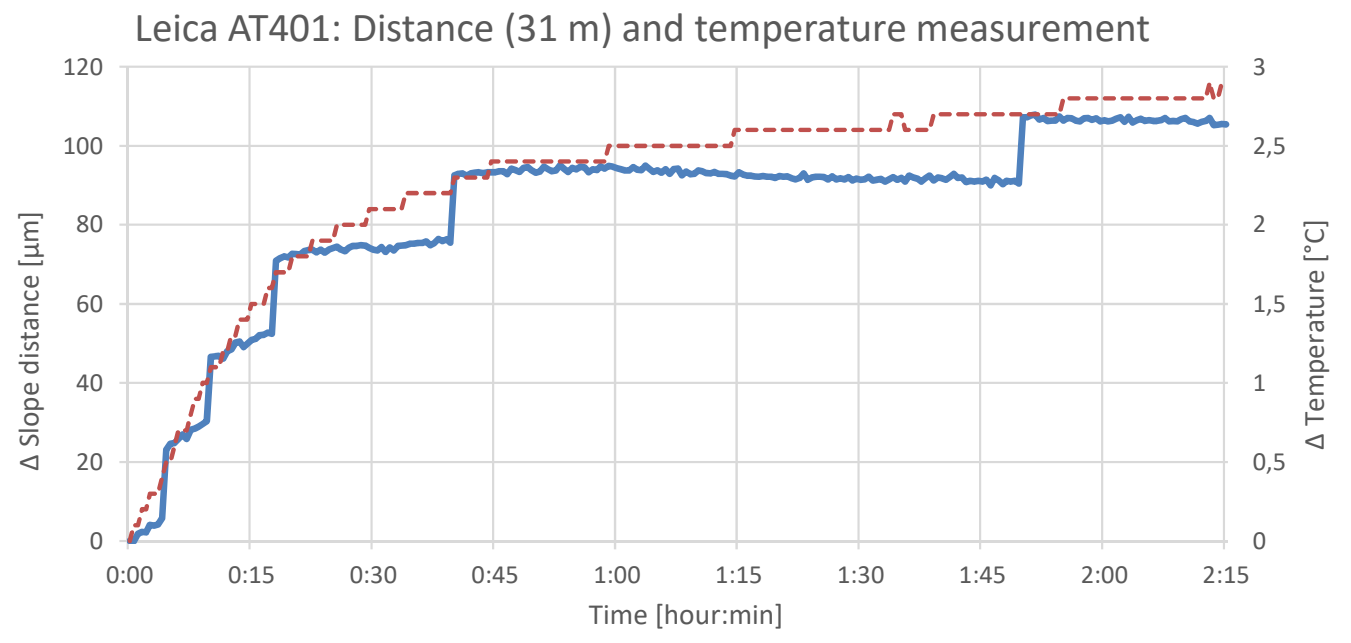

FiguRE 4. Improper updates of the refractive indices at the Leica AT401.

\begin{tabular}{|c|r|r|r|r|r|r|}
\hline \multirow{2}{*}{ Error in } & \multicolumn{2}{|c|}{$\begin{array}{c}\text { Extreme cond. } \\
(\mathbf{1 6 0} \mathbf{~})\end{array}$} & \multicolumn{4}{|c|}{ Ordinary conditions } \\
\cline { 2 - 7 } & \multicolumn{1}{|c|}{ Min } & \multicolumn{1}{c|}{ Max } & \multicolumn{1}{c|}{ Min } & Max & \multicolumn{1}{c|}{ Min } & \multicolumn{1}{c|}{ Max } \\
\hline Ref. model & 2 & 102 & 2 & 4 & 2 & 51 \\
\hline Wavelength & -50 & -43 & -8 & -8 & -45 & -43 \\
\hline Update of $\boldsymbol{n}$ & -80 & 80 & -8 & 8 & -80 & 80 \\
\hline Total & -128 & 139 & -14 & 3 & -123 & 88 \\
\hline
\end{tabular}

TABLE 2. Errors of Leica AT401 system software $[\mu \mathrm{m}]$.

only if the new computed value differs at least $0.5 \mathrm{ppm}$ from the old one. This behaviour is not acceptable when measurements are performed in changeable conditions.

Another fact, which influences the measurement, is that temperature readings are stored with $0.01{ }^{\circ} \mathrm{C}$ resolution at temperatures below $10^{\circ} \mathrm{C}$ (e.g., $9.99^{\circ} \mathrm{C}$ ), but only with $0.1^{\circ} \mathrm{C}$ resolution at temperatures above $10^{\circ} \mathrm{C}$ (e.g., $\left.10.1^{\circ} \mathrm{C}\right)$. These errors effect both the absolute correctness and the relative continuity of measurement (Fig. 4)

The effect of the errors, which in many cases exceeds instrument's accuracy specifications, is shown in Table 2. The presented errors were found in three different system software versions. Ever since then no information about fixing these issues was published in "What's new" documentations by Leica. As all AT40x instruments share the same system software package, it is highly probable that also AT402 and AT403 devices are affected in the same way. It was impossible to negotiate a proper solution with the manufacturer.

All this, found at the exceptionally accurate instrument from a well-known manufacturer, is disturbing and signifying the need for users to orientate in the field of the refractive index of air. In this particular case, all errors of Leica AT401 system software, except for the changeable temperature resolution issue, could be eliminated by developing and using a user-programmed controlling software called ATControl 22 .

\section{Conclusions}

A detailed overview of 10 existing methods of indirect computation of the group refractive index of air was given in this paper. Wide intervals of wavelengths and atmospheric parameters were used to test all chosen methods with respect to the most advanced procedure developed by Ciddor and Hill. It proved that some equations, which are still frequently used in geodesy, are obsolete and should not be used for measurements in engineering geodesy and metrology. Differences up to $0.5 \mathrm{ppm}$ were found even for wavelengths used in EDM instruments integrated in geodetic total stations.

As a result, a complex procedure developed by Ciddor and Hill in 1999 was chosen and adapted to be used for accurate measurements. such as the calibration of the Czech State Long Distances Measuring Standard Koštice and National Baseline Hvězda using the Leica AT401 laser tracker. For ordinary distance meters used in geodesy, the simplified method according to the resolution of International Association of Geodesy from 1999 in Birmingham is sufficiently accurate because it is in congruence with the precise method at about 0.1 ppm level.

In the end, the Ciddor and Hill procedures were considered to be updated with newer equations and constants for air density according to CIPM-2007. However, it was experimentally tested that the update would not produce a change in results which would be significant in practice. The example case of the Leica AT401 showed that manufacturers do not always cover the topic of the refractive index of air properly. If users want to control the entire process of obtaining distances from their instruments, they should be interested in how the first velocity corrections are automatically computed by the firmware/software.

\section{ACKNOWLEDGEMENTS}

This work was supported by SGS18/055/OHK1/1T/11 grant by the Czech Technical University in Prague. 


\section{REFERENCES}

[1] H. Barrell, J. E. Sears. The refraction and dispersion of air for the visible spectrum. Philosophical Transactions of the Royal Society A: Mathematical, Physical and Engineering Sciences 238(786):1-64, 1939. DOI:10.1098/rsta.1939.0004.

[2] Y. Yamaoka, K. Minoshima, H. Matsumoto. Direct measurement of the group refractive index of air with interferometry between adjacent femtosecond pulses. Applied Optics 41(21):4318-4324, 2002. DOI:10.1364/AO.41.004318

[3] A. L. Buck. Model cr-1a hygrometer with autofill, 2012.

[4] C. Burnside. Electromagnetic distance measurement. Collins, London, 2nd edn., 1982.

[5] J. M. Rueger. Electronic Distance Measurement. Springer-Verlag, Berlin, 1990.

[6] B. Edlén. The dispersion of standard air. Journal of the Optical Society of America 43(5):339-344, 1953. DOI:10.1364/JOSA.43.000339

[7] B. Edlén. The refractive index of air. Metrologia 2(2):71-80, 1966

[8] J. C. Owens. Optical refractive index of air. Applied Optics 6:51-59, 1967. DOI:10.1364/AO.6.000051.

[9] E. R. Peck, K. Reeder. Dispersion of air. Journal of the Optical Society of America 62(8):958-962, 1972. DOI:10.1364/JOSA.62.000958

[10] K. P. Birch, M. J. Downs. An updated edlén equation for the refractive index of air. Metrologia 30:155-162, 1993.

[11] K. P. Birch, M. J. Downs. Correction to the updated edlén equation for the refractive index of air. Metrologia 31:315-316, 1994.

[12] P. E. Ciddor. Refractive index of air: new equations for the visible and near infrared. Applied Optics 35(9):1566-1573, 1996. DOI:10.1364/AO.35.001566
[13] P. E. Ciddor, R. J. Hill. Refractive index of air: 2 group index. Applied Optics 38(9):1663-1667, 1999. DOI:10.1364/AO.38.001663

[14] P. E. Ciddor. Refractive index of air: 3. the roles of $\mathrm{CO} 2, \mathrm{H} 2 \mathrm{O}$, and refractivity virials. Applied Optics 41(12):2292-2298, 2002. DOI:10.1364/AO.41.002292

[15] P. E. Ciddor. Refractive index of air: 3. the roles of $\mathrm{CO} 2, \mathrm{H} 2 \mathrm{O}$, and refractivity virials: erratum. Applied Optics 41(33):7036-7036, 2002. DOI:10.1364/AO.41.007036

[16] F. Dvoracek. CIDDOR \& HILL - 1996, 1999. http: //k154.fsv.cvut.cz/ dvoracek/doc/ciddor.pdf [2018-02-01].

[17] P. Giacomo. Equation for the determination of the density of moist air (1981). Metrologia 18(1):33-40, 1982.

[18] R. S. Davis. Equation for the determination of the density of moist air (1981/91). Metrologia 29(1):67-70, 1992.

[19] A. Picard, R. S. Davis, M. Gläser, K. Fujii. Revised formula for the density of moist air (cipm-2007). Metrologia 45(2):149-155, 2008. DOI:10.1088/0026-1394/45/2/004.

[20] National Institute of Standards and Technology. Engineering Metrology Toolbox. http://emtoolbox.nist.gov/Wavelength/Ciddor.asp [2018-02-01].

[21] F. Dvořáček. Laboratory testing of the leica at401 laser tracker. Acta Polytechnica 56(2):88-98, 2016. DOI:10.14311/AP.2016.56.0088.

[22] F. Dvořáček. Atcontrol: Controlling software for leica at40x laser trackers. Geoinformatics FCE CTU 14(2):9-20, 2015. DOI:10.14311/gi.14.2.2 